Arthroskopie 2021 · 34:331-332

https://doi.org/10.1007/s00142-021-00492-5

Angenommen: 28. September 2021

Online publiziert: 30 . September 2021

( $)$ Springer Medizin Verlag $\mathrm{GmbH}$, ein Teil von

Springer Nature 2021

\section{Nachruf auf Freddie Fu}

\section{0-2021}

Andreas B. Imhoff

Abteilung für Sportorthopädie, Klinikum rechts der Isar, Tech. Universität München (TUM), München, Deutschland

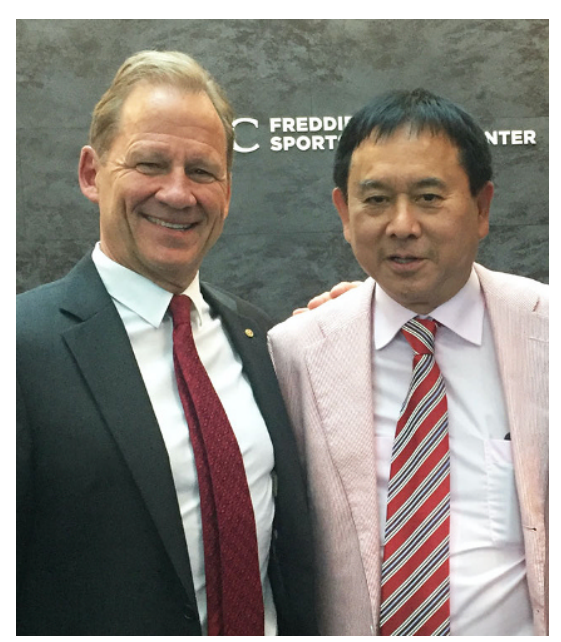

Wir trauern um Freddie Fu, einem langjährigen Ehrenmitglied der AGA, der am Freitagabend, den 24.09.2021, 70-jährig nach kurzer Krankheit gestorben ist. Er wurde in den Wochen vor seinem Tod wegen einem malignen Melanom mit Nierenblutungen behandelt.

Freddie war ein Pionier der orthopädischen Chirurgie, Gründer des „Sports Medicine Program" an der University of Pittsburgh Medical Center (UPMC), eine weltbekannte sportorthopädische Ausbildungsschmiede. Seine immense Expertise machte ihn zum bislang anerkanntesten Wissenschaftler in der orthopädischen Sportmedizin mit über 60.000 Zitaten.

Er erhielt weltweite Anerkennung für seine Erfindungen, Op.-Techniken und Behandlungsmöglichkeiten, v. a. am Kniegelenk und bei vielen Sportverletzungen. Selbst als Chairman des Dept. of Orthopedic Surgery berufener David-Silver-Professor of orthopedic surgery at Pitt's School of Medicine widmete er sich nicht nur den Spitzenathleten sondern nahm sich auch Zeit für alle anderen Patienten. Ein wichtiges Anliegen war die Ausbildung von
Studenten, Fellows und jungen Ärzten zu jeder Tages- und Nachtzeit.

Ich hatte die unglaubliche Gelegenheit als erster 1-Jahres-Fellow der AGA 1994/95 bei ihm arbeiten und lernen zu dürfen. Trotz der vielen Fellows aus allen Ecken der Welt hat er mich von Beginn an voll akzeptiert. In den vielen intensiven Diskussionen zu Forschung, Lehre und Krankenversorgung hat sich ein intensives freundschaftliches Verhältnis über die Jahre weiterentwickelt. Ich habe seine Menschlichkeit, Gastfreundschaft und Großzügigkeit, aber auch seine Organisationsfähigkeit im Management eines großen orthopädischen Departments mit intensiver Forschungstätigkeit bewundert und meine Leitungs- und Managementerfahrungen mit ihm austauschen können.

Sein außerordentlich menschlicher Charakter widerspiegelte sich auch in der Integration der Familien seiner Fellows. So nahm er einmal auf dem Weg zu einem Sporttrainingscamp meine 3 damals kleinen Söhne Florian, Pascal und Dominik mit kindlicher Freude in den McDonalds mit und verwöhnte sie über alle Maßen.

Freddie wuchs in Hong Kong auf, war bereits in der Grundschule sportlich sehr aktiv mit Schwimmen und Basketballspielen, bevor er 1970 als 18-Jähriger nach Pittsburgh kam. Dr. Albert Ferguson, der damalige Chairman des orthopädischen Departments, entdeckte inn während des Medizinstudiums, motivierte ihn und wurde über viele Jahrzehnte sein Mentor. Nach dem Studium wurde mit einem orthopädischen Forschungsfellowship unter Ferguson der Startschuss zu seiner späteren Karriere gegeben.

Während meiner Zeit in Pittsburgh widerstand Freddie einem Abwerbeversuch an die University of Pennsylvania in Phi- 
ladelphia und entschied sich mit seiner ganzen Energie in Pittsburgh ein sportmedizinisches Department aufzubauen. Der Forschungsschwerpunkt fokussierte er auf das vordere Kreuzband, was in den folgenden Jahrzehnten zu einer immensen Zahl von Publikationen in Biologie, Biomechanik, Op.-Techniken und Rehabilitation geführt hatte. So wurde er auch gerne in Anlehnung an die "Double-Bundle-Kreuzbandtechnik" als "Double Bundle of Energy Surgeon" bezeichnet. Die Forschungen seines Teams sind weltweit führend und wegweisend, so dass er und sein Team unzählige Ehrungen und Preise zugesprochen bekam. Seine tägliche, manchmal 24stündige Motivation aus klinischen Fragestellungen direkt Forschungsergebnisse zu fördern, hatte auch mich in all den Jahren in München beflügelt. Wir haben ein Austauschprogramm der AGA mit UPMC seit 1994 intensiv gepflegt, das wir hoffentlich auch mit Prof. Volker Musahl weiterführen dürfen. Wir haben viele gemeinsame Forschungen und Projekte durchführen und auch gemeinsame Bücher, wenn auch manchmal zur Schulterchirurgie (!), herausgeben dürfen.

Ich werde Freddie mit meiner Familie, meiner Frau Susan und meinen 3 Kindern als meinen wichtigsten Mentor nie vergessen und bin unendlich traurig für diesen jähen Verlust.

München, 27.09.2021

Univ. Professor Andreas B. Imhoff

\section{Korrespondenzadresse}

Univ. Prof. Dr. med. Andreas B. Imhoff Abteilung für Sportorthopädie, Klinikum rechts der Isar, Tech. Universität München (TUM) Ismaninger Str. 22, 81675 München, Deutschland imhoff@tum.de
Hier steht eine Anzeige.

黛 Springer 